\title{
Contextual Inquiry in Signal Boxes of a Railway Organization*
}

\author{
Joke Van Kerckhoven, Sabine Geldof, and Bart Vermeersch \\ Namahn, Grensstraat 21, 1210 Brussels, Belgium \\ $\{j v k e, s g, b v\} @$ namahn. com
}

\begin{abstract}
A number of selected field-study techniques have been validated in a case study in the domain of railway signal boxes. The context of this work is the endeavour of a human-centred design consultancy to acquire know-how on HCI methods for use in the design of safety-critical systems. The field studies were aimed at providing a clear overview of the work environment, tasks and cognitive load of the signallers and of possible bottlenecks in the current way of operating. Our approach for conducting field studies in supervisory control systems is based on ethnomethodology, situation awareness, and mental models represented as an abstraction hierarchy. For each of these methods, we discuss our approach, the result, and the applicability of the technique to future safetycritical system design projects.
\end{abstract}

Keywords: Safety-critical systems, ethnomethodology, patterns of cooperative interaction, situation awareness, goal-directed task analysis, mental models, abstraction hierarchy.

\section{Introduction}

As the practice of human-centred design gains more ground, also in the domain of safety-critical systems, it is important to know which methods for field studies can yield the most practically useful results in specific settings. Safety-critical systems have stringent requirements in terms of error avoidance, efficiency and risk management. Performance errors are serious because of their enormous impact on human health or the environment. Typical applications include traffic control, process supervision, critical care and emergency response.

As a human-centred design consultancy involved in designing for safety-critical systems, we want to increase our internal know-how on interaction design for these systems, i.e. specialist knowledge, techniques and expertise. We conducted a research project funded by the Institute for the Encouragement of Scientific Research and Innovation of Brussels (IRSIB) ${ }^{1}$. In the context of this project, we performed field studies in railway signal boxes [1]. This paper describes our experience in validating a

\footnotetext{
The original version of this chapter was revised: The copyright line was incorrect. This has been corrected. The Erratum to this chapter is available at DOI: 10.1007/978-3-642-11750-3_10

* The research described is funded by IWOIB/IRSIB, the Institute for the Encouragement of Scientific Research and Innovation of Brussels (project reference: RBC/06 R-144).

${ }^{1}$ More information about the activities of IWOIB/IRSIB can be found on http://www.iwoib.irisnet.be/index_en.htm

P. Palanque, J. Vanderdonckt, and M. Winckler (Eds.): HESSD 2009, LNCS 5962, pp. 96-106, 2010.

(C) Springer-Verlag Berlin Heidelberg 2010
} 
number of research techniques in a case study and the conclusions drawn from this activity. In section 2 we briefly describe the context of our work, i.e. the larger research project. Section 3 introduces the practical context: the case study in which we performed the field study. Sections 4, 5 and 6 describe each of the validated frameworks. Our conclusions are presented in section 7 .

\section{Research Project on Methodology for Safety-Critical Systems}

The goal of the research project was to acquire know-how about selected Models, Theories and Frameworks [2] ('MTFs') that might be useful in interaction design for safety-critical systems and in translating that knowledge into practice-oriented and market-worthy methods. To this end, we adopted a three-step process and applied it to a number of MTFs.

\subsection{Hypothesis}

Candidate MTFs were assessed on their supposed relevance to the domain of safetycritical systems. Figure 1 positions the selected MTFs with regards to the social dimension (whether the individual user or the larger environment is concerned) and to the human-centred design process. The following MTFs were the object of our study: Ethnomethodology (ETHNO), Distributed Cognition (DC), Human Decision Making (HDM), Mental Modelling (MM), Cognitive Work Analysis and Ecological Interface Design (CWA/EID), Human Visual Perception (PERC), Motor Behaviour Models (MOTOR). For each MTF we explored existing literature and devised hypotheses about the applicability of the MTF. These hypotheses addressed the question "How could we use this theoretical knowledge when designing for safety-critical systems?"

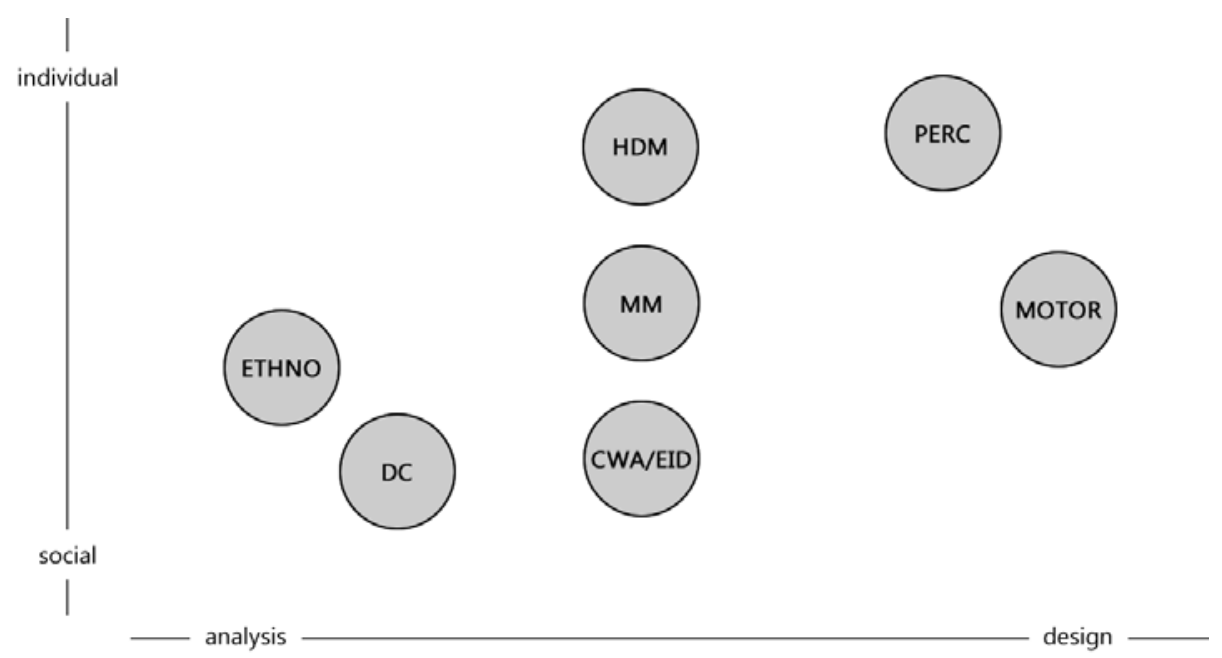

Fig. 1. Overview of the MTFs studied in the research project 


\subsection{Validation via Case Studies}

Our understanding of these hypotheses and their value to the practice of safetycritical systems design were validated through realistic case studies in four different domains:

- Case 1: Diagnosis of liver cancer

- Case 2: Decision making for maxillofacial surgery

- Case 3: Configuration of ground-based satellite equipment

- Case 4: Supervisory control of railway signalling equipment (the subject of this paper)

Based on the outcome of the literature study and the type of case studies at hand, a number of hypotheses were assigned to each case, as shown in Figure 2.

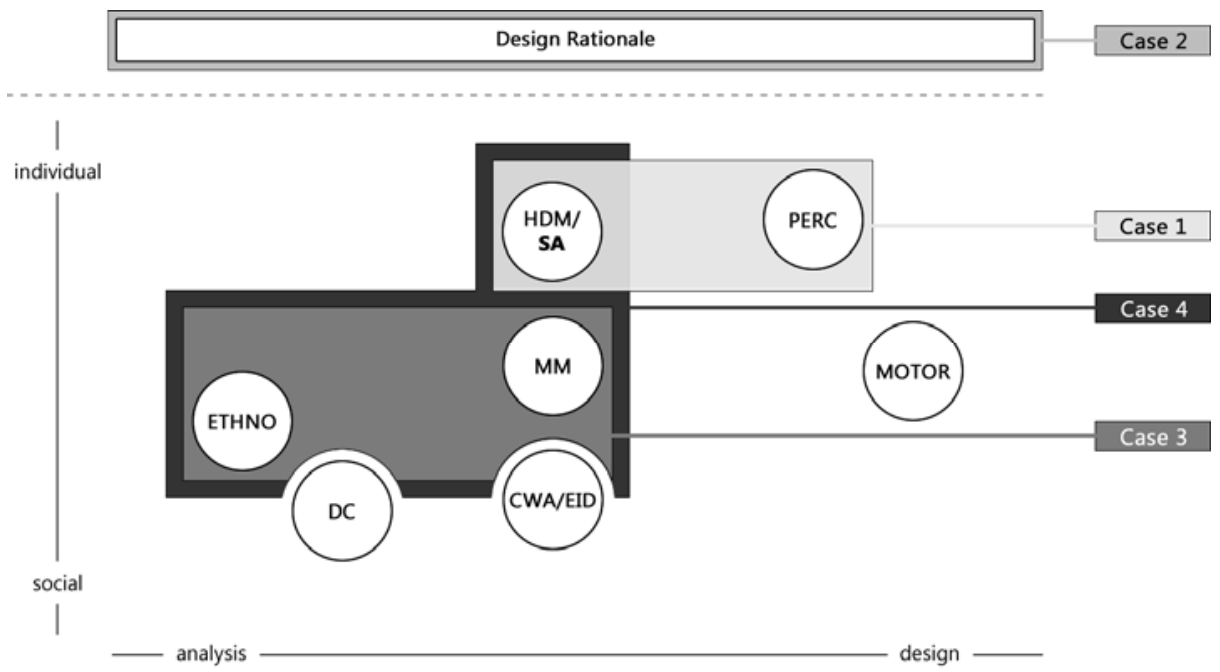

Fig. 2. Overview of the MTFs validated in the cases

In Case 4 (discussed in this paper) we validated three MTFs, two of which (Ethnomethodology and Mental Modelling) were also validated in another case. The third MTF, Situation Awareness (SA) was selected because the application type of this case study is 'supervisory control'. We considered it useful to study this important aspect of human decision making in safety-critical systems.

\subsection{Consolidation}

The lessons learned from the validation step were translated into methodology components. For each component, we provided a definition, a roadmap, resources and applicability criteria. 


\section{Case Study: Supervisory Control of Railway Signalling Equipment}

This case study focused on the work of signallers in the signal boxes of a railway operator. From this control room, signallers manage the switches and signals of the railway infrastructure in a certain geographical area, comprising different action zones and sectors. The signal boxes we studied are equipped with a modern computer-based control system. Most routine jobs are automated, but interventions are required on a regular basis. The work is distributed as follows:

- An operator controls the signals and switches of a given action zone;

- A sector manager leads the operators working in his sector, which corresponds to a number of action zones;

- A zone regulator is in charge of the entire working zone of the signal box.

The workstations are grouped per sector and organized in two or three rows, as shown in Figure 3. In addition to displays on the desks, there is a large control display at the front of the room.
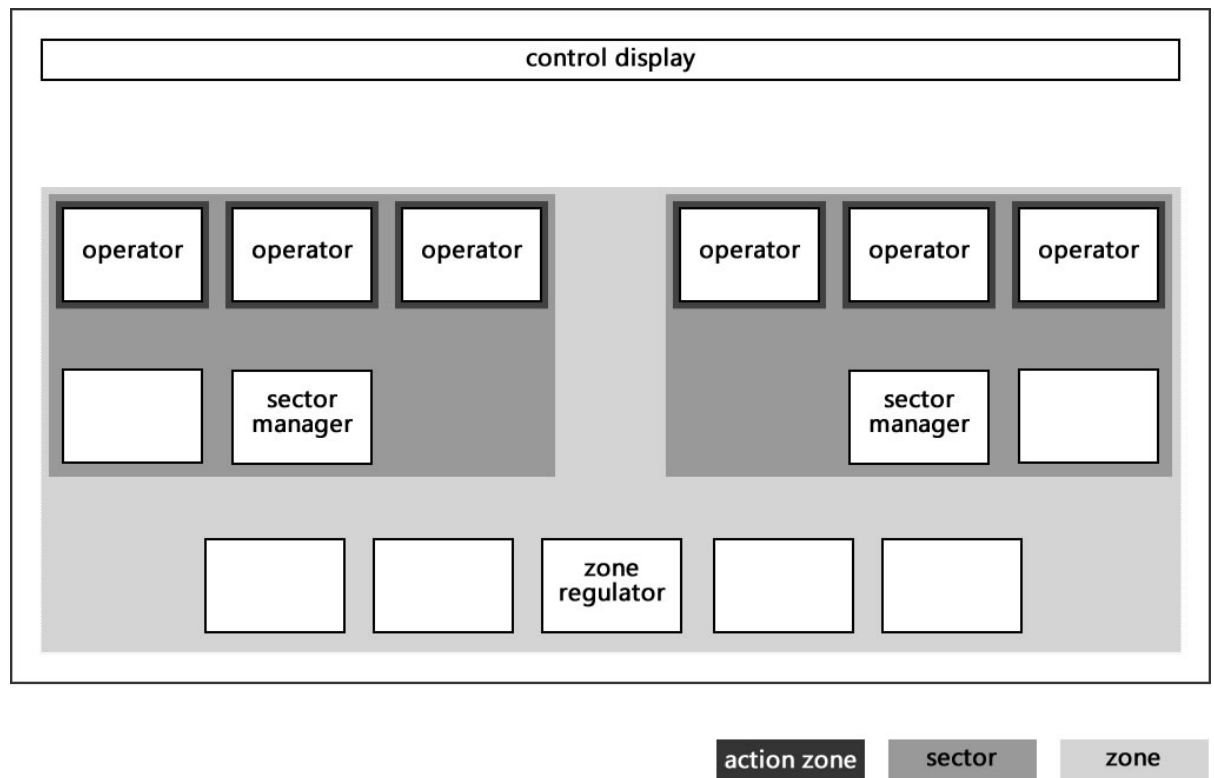

Fig. 3. Typical organization of a signal box

The aim of our research was to obtain a clear understanding of the cognitive and socio-technical dimensions of the work through field studies. So far, we have observed two operating signal boxes for five days in total. In order to structure and focus our field observations, we used the technique of ethnomethodology and insights from our previous study of situation awareness and mental modelling. As shown in 
Figure 4, these MTFs complement each other in that they each deal with another aspect of the work:

- Ethnomethodology focuses on co-operation between the different actors in the control room.

- Situation awareness is the awareness a person needs to have about system events in order to make correct decisions.

- Mental modelling elicits the internalized image a user applies to mentally simulate and reason about the behaviour of a system.

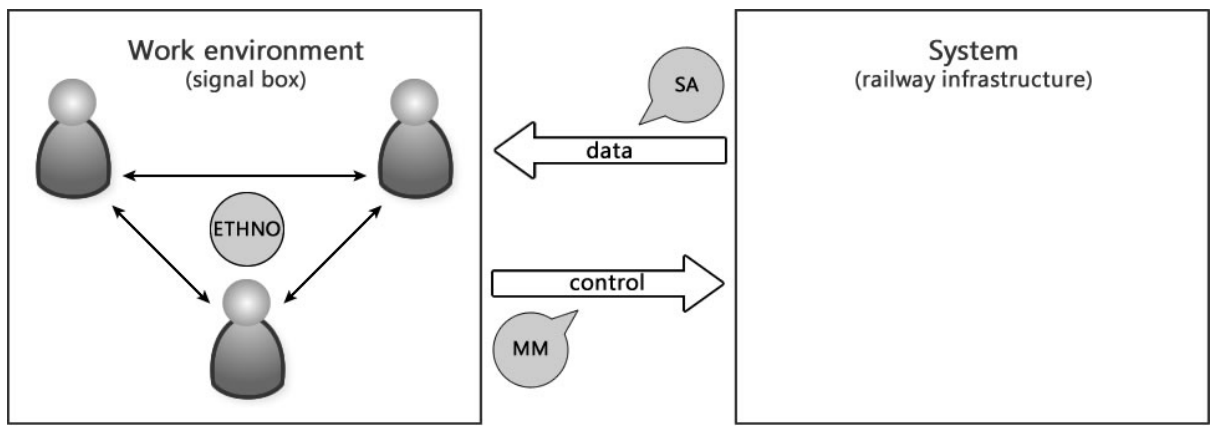

Fig. 4. The complementary MTFs applied in the case study

\section{Ethnomethodology}

Ethnomethodology is a sociological discipline that studies how social order is achieved. It assumes that people construct their social order in the process of acting together.

In HCI, ethnomethodology is mainly used as a model for analyzing work. Officially, work is organized by formal tasks, processes and procedures, but this is only in theory. In practice, workers need to get the job done, regardless of the way their work is organized on paper.

Also in the work environments of safety-critical systems, work relies on tacit practices and informal communication. Attempting to formalise these practices would provide an unreliable foundation for the design of systems to support co-operative work [3].

By using ethnomethodology, we tried to grasp the tacit knowledge and informal communication that is not explicitly described in the official work instructions or task descriptions.

\subsection{Approach: Patterns of Cooperative Interaction}

Traditional ethnomethodological analyses are time consuming. Several months are needed for observations, analysis, and interpretation. Furthermore, the results are specific to a certain working environment and certain situations, so they are not reusable.

However, Martin and Sommerville [4] identified and described 10 patterns of cooperative interaction from previous ethnomethodological studies that can be 
generalized and reused. The patterns are defined as 'regularities in the organisation of work, activity, and interaction amongst participants, and with, through and around artefacts'. The pattern descriptions are easily accessible, even for analysts or designers who lack the expertise of an ethnographer.

Most of these patterns are not specific to safety-critical systems, but they are recognized as important in field studies for systems design [4]. We used them as a focal point during our observations, and structured our findings according to them.

\subsection{Result}

Collaboration in small groups is one of the patterns we identified when observing the signallers' work. This pattern is concerned with the manner in which small, colocated groups collaborate to carry out tasks. The pattern draws attention to the way in which collaboration is facilitated by seating arrangements and various artefacts. [4]

In signal boxes, a sector manager and his operators cooperate intensively in order to be able to efficiently respond to the situation. They continuously shout to each other to exchange information quickly. The acoustics of the signal box is thus a crucial factor affecting the success of the collaboration.

We found that the usual seating arrangement in signal boxes is not ideal. There is no visual contact between a sector manager and his operators, which hinders communication. One is not able to visually verify whether the other party has successfully received a message. Furthermore, the sector manager is not able to see what the operators are doing.

The large control display at the front of the signal box is a typical example of the pattern public artefact. The display should facilitate communication, but unfortunately, it is hardly ever used. The information displayed shows insufficient detail, whereas a complete overview of the working zone of the signal box is necessary for decision making. In addition, the set-up of the signal boxes makes it impossible for signallers to see the full screen: the display doesn't fit into the field of vision from the first or second row of seats. Small font sizes make the information on the display illegible.

The patterns described above are only two examples of patterns we recognized. It turns out that all patterns listed by Martin and Sommerville were applicable to the signallers' work in some way.

\subsection{Evaluation}

The patterns of cooperative interaction provide a valuable framework for conducting field studies within a limited budget and timeframe. They offer a useful guide to structure observations themselves as well as the subsequent reporting.

We expected that using the patterns would later help us to optimally gear the design of an application to the context of use. However, making the link between analysis and design remains a creative step. The patterns don't include answers to the following questions:

- What are the design consequences if a particular pattern is observed?

- What is the relationship between the patterns, for example, can they compete?

- Are some patterns preferred over others? 
- Do some patterns indicate a bad practice which should be eliminated?

- Are there strongly recommended patterns that should therefore be installed by design?

In addition, we discovered that it would be useful to enrich each pattern with focal points, pitfalls or guidelines that one should take into account during observation.

\section{Situation Awareness}

Endsley, Bolte and Jones [5] describe Situation Awareness (SA) as "being aware of what is happening around you and understanding what that information means to you now and in the future'. In control rooms, situation awareness is a human skill that arises, amongst others, through the peripheral awareness of users interacting with a variety of information sources (e.g. monitoring screens, colleague interactions, interaction with other devices) [6]. In our study, we focused on eliciting the information that is relevant for performing a particular task.

In work situations, situation awareness is closely related to the goals and objectives associated with a specific job. It is especially important for effective decision making, and therefore a crucial factor in safety-critical systems.

We wanted to investigate how situation awareness can be promoted in the signallers' work situation.

\subsection{Approach: Goal-Directed Task Analysis}

We validated goal-directed task analysis (GDTA), as suggested by Endsley, Bolte and Jones [5], as a method for examining what information signallers need to perform their job. GDTA seeks to discover the user's information needs for optimally achieving his primary goals and making well-founded decisions.

Our research focused on the information needs of the sector manager:

- What are the goals a sector manager is trying to achieve?

- Which decisions need to be made for attaining these goals?

- What information would a sector manager ideally need to know for making these decisions?

These questions couldn't be answered directly by signallers, but had to be elicited via interviews and observations during real-time operations. The information gathered was organized into a hierarchy of goals, sub goals, decisions and situation awareness requirements.

\subsection{Result}

The overall goal of a sector manager was defined as follows:

- Guarantee the safety of railway traffic.

- Guarantee the timing of railway traffic.

- Guarantee the comfort of passengers.

One of the sub goals classified under this last goal is 'give priority to trains that are vital links for passenger traffic'. The related decision and situation awareness requirements are illustrated in Figure 5. 


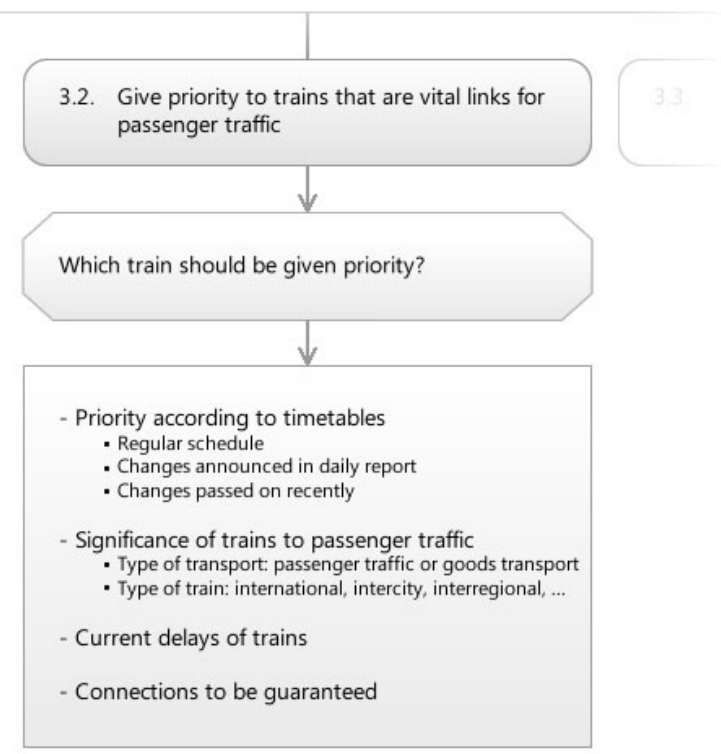

Fig. 5. Extract from the goal hierarchy of a sector manager

\subsection{Evaluation}

GDTA turned out to be a useful method to determine the information a user needs for making decisions. It is not easy to think in terms of goals instead of tasks, but in this case study, it proved to be a valuable method to uncover the information needs in a structured way, i.e. linked to the goal structure.

Furthermore, the focus on goals revealed an important difficulty which complicates the decision making process of a sector manager. It became clear that the three goals of the sector manager are contradictory. For example, raising the degree of safety has a negative effect on timing and vice versa. Therefore, a sector manager often struggles with making the right decisions: which goal should be given priority?

The given timeframe didn't allow us to create a complete goal hierarchy. In order to gain complete insight into the information needs, a more thorough analysis is required. This not only requires more observation time, but also evaluation of the analysis result (a GDTA structure) by users in several iterations.

\section{Mental Models}

To cope with problems arising in the control of complex systems, an operator has to address each problem at the appropriate level of cognitive resolution: some problems require a detailed view of the information, while for others, an overview is more appropriate so as to not make the problem unnecessarily complex.

We aimed to develop a mental model of the railway infrastructure that would help signallers structure the situation at several levels of complexity. 


\subsection{Approach: Abstraction Hierarchy}

We wanted to verify whether the abstraction hierarchy, as proposed by Rasmussen [7], provides a good framework for representing a mental model of complex systems like a railway infrastructure.

Rasmussen suggests structuring a mental model on five levels of decreasing abstraction in a functional hierarchy:

- Functional purpose: system objectives and constraints

- Abstract function: the causal structure (physical laws, etc.)

- Generalized function: general functions and processes

- Physical function: physical functions and processes of the components

- Physical form: the material configuration of the system.

Structuring the system according to these levels helps reasoning about problems: upwards to a reason, and downwards to a cause. Within the domain of railway transportation, this framework has been used to create a macrocognitive representation model in view of coordinating various human factors projects and efforts within the field [8].

We attempted to pick up the mental model used by signallers via observations and interviews during real-time operations, and to visualize that model by means of an abstraction hierarchy.

\subsection{Result}

The result of our effort to create a layered mental model is shown in Figure 6. Note that this mental model is incomplete, because we encountered several difficulties as described below.

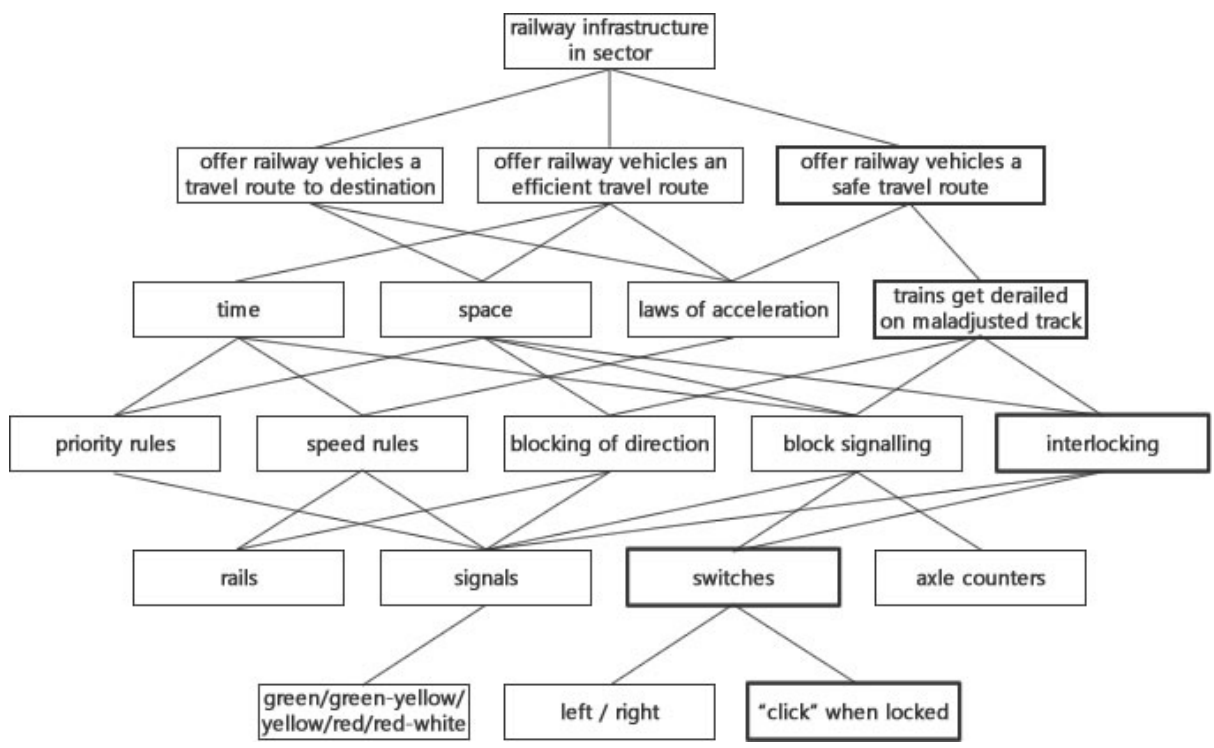

Fig. 6. (Incomplete) mental model of the railway infrastructure in five levels of abstraction 
In the above diagram, several causal paths lead from the functional purpose of the system (top level) to a physical form (bottom level). Let's consider one of the paths as an example (the boxes with a darker outline).

'Offer railway vehicles a safe travel route' is one of the system objectives. To guarantee safety, one must take into account that trains can derail on a maladjusted track. 'Interlocking' is a typical process that ensures trains stay on the track: all switches on the desired route first have to be aligned and locked mechanically, before the train receives permission to depart. Switches are the physical components linked with interlocking. On a physical level, the locking of a switch manifests itself via an audible 'click'.

Following the path in the opposite direction, one can trace the link from a physical manifestation all the way up to a functional purpose of the system.

\subsection{Evaluation}

As human-centred designers, we feel the need to provide the user with an understandable system representation, which enables him to align his mental model with reality. However, we found that the mental model according to an abstraction hierarchy wasn't appropriate for this purpose.

Firstly, the mental model remains too abstract, because it lacks context and doesn't characterize important relationships, e.g. between the elements of one abstraction level. Also, in contrast with a traditional mental model, an abstraction hierarchy contains concepts about which a user is not or should not be aware. In this perspective, the abstraction hierarchy is more closely related to a business domain than a user's mental model. The result is that this mental model wouldn't succeed in supporting the user when interacting with the system.

Secondly, even though we have not yet completed the mental model, its complexity already has a degrading effect on the readability of the diagram. We suspect that completing the mental model would make this diagram unmanageably large and complex.

In addition, creating a good mental model representation according to an abstraction hierarchy is far from obvious. An in-depth understanding of the system is required. Since the mental model adds little value for communication with the users, the effort may not be justified.

For these reasons, we doubt the usefulness of the abstraction hierarchy for representing the mental model of signallers. However, others [8] found the framework useful in creating a high-level integrative model of railway operations to coordinate the efforts of a human factors team.

\section{Conclusion}

The aim of this project was to find out whether the selected MTFs are appropriate for field studies in safety-critical systems. Based on our field study in the signal boxes, we can draw the following conclusions:

- Patterns of cooperative interaction are useful for structuring observations. However, elaborating the specific characteristics for each pattern would significantly increase the added value. 
- Goal-directed task analysis is a useful method to determine the correct information a user needs for making decisions, and to uncover conflicts between the goals one tries to achieve when making these decisions. However, we feel that user feedback on the resulting hierarchy is required.

- The added value of a mental model based on an abstraction hierarchy appeared small, certainly when compared to the effort it took to build it. Further investigation is needed to determine the circumstances in which an abstraction hierarchy would be appropriate.

We are already applying the know-how acquired through this research project to human-centred design projects for safety-critical systems.

With respect to our field study in the signal boxes, we hope to continue our analysis in order to elaborate our findings and offer useful advice for improving the working conditions of signallers.

\section{References}

1. Geldof, S., Van Kerckhoven, J.: Field study techniques for supervisory control systems. In: Drury, J. (ed.) Proceedings of the HCP-2008 Workshop on Supervisory Control in Critical Systems Management, Delft (2008)

2. Carroll, J.M. (ed.): HCI Models, Theories and Frameworks. Morgan Kaufmann, San Francisco (2003)

3. Heath, C., Luff, P.: Technology in action. Cambridge University Press, Cambridge (2000)

4. Martin, D., Sommerville, I.: Patterns of Cooperative Interaction: Linking Ethnomethodology and Design. ACM Transactions on Computer-Human Interaction 11(1), 59-89 (2003)

5. Endsley, M.R., Bolte, B., Jones, D.J.: Designing for situation awareness: An Approach to User-Centered Design. Lawrence Erlbaum Associates, NJ (2003)

6. Luff, P., Heath, C.: Naturalistic analysis of control room activities. In: Noyes, J., Bransby, M. (eds.) People in control. IEE control engineering series, pp. 151-167. The Institution of Electrical Engineers, London (2002)

7. Rasmussen, J.: The role of hierarchical knowledge representation in decision making and system management. IEEE Transactions on Systems, Man and Cybernetics 15, 234-243 (1985)

8. Bye, R., Farrington-Darby, T., Cox, G., Hockey, G.R.J., Wilson, J.R., Clarke, T.: Work Analysis and Distributed Cognition Representation of Integrated Rail Operations. In: Wilson, J., Norris, B., Clarke, T., Mills, A. (eds.) People and Rail Systems, pp. 275-283. Ashgate, Aldershot (2007) 